\title{
ANÁLISIS DE LA FUNCIÓN DE CORRECCIÓN DE LA VELOCIDAD DE SEDIMENTACIÓN PARA MICRO PARTÍCULAS
}

\section{ANALYSIS OF THE CORRECTION FUNCTION FOR MICRO-PARTICLE SEDIMENTATION VELOCITY}

\author{
Gonzalo Salinas-Salas $^{1} \quad$ Igor Ruiz-Tagle-Gutiérrez $^{2} \quad$ Frank Babick $^{3}$ \\ Recibido 6 de diciembre de 2006, aceptado 13 de septiembre de 2007 \\ Received: December 6, $2006 \quad$ Accepted: September 13, 2007
}

\begin{abstract}
RESUMEN
La velocidad de sedimentación de las partículas presentes en una suspensión sufre una caída monótona en función de la concentración volumétrica de éstas, por efecto de las fuerzas hidrodinámicas y electroquímicas que se presentan en una suspensión. El valor efectivo que alcanza la velocidad de sedimentación puede evaluarse a partir de la velocidad de sedimentación teórica de una partícula única, multiplicada por la denominada función de corrección de velocidad o función obstáculo, la que considera tanto el régimen de escurrimiento como la concentración volumétrica de partículas. Los valores determinados para esta función por Richardson y Zaki en 1954 [14] son los más utilizados actualmente, donde el valor propuesto para el caso de regímenes de escurrimiento del fluido por sobre las partículas, cuyos números de Reynolds sean menores a 0,25 , se establece un valor único de 4,65, independientemente del tamaño de las partículas. El presente artículo muestra los resultados alcanzados a partir de un trabajo experimental desarrollado con micro partículas calibradas de óxido de silicio $\left(\mathrm{SiO}_{2}\right)$, que indica que el valor del exponente de la función de corrección depende inversamente del tamaño, para el caso de partículas de orden micrométrico, lo que daría lugar a un nuevo valor para el exponente.
\end{abstract}

Palabras clave: Función de corrección, velocidad de sedimentación, micro partículas.

\section{ABSTRACT}

The sedimentation velocity of micro-particles in suspension decreases with increasing concentration due to hydrodynamic and electrostatic forces. This velocity can be estimated on the basis of the theoretical velocity of a single particle, multiplied by a correction factor which depends on the flow regime as well as the volumetric concentration of the particles. The most commonly used values are those determined by Richardson and Zaki in 1954 [14]. For flow regimes characterized by a Reynolds' number less than 0,25, a constant value of 4,65 is used which does not depend on the particle size. The present article presents results of micro-particle sedimentation research performed with calibrated silicon oxide particles $\left(\mathrm{SO}_{2}\right)$, which indicate that the exponent in the correction function is inversely dependent on micro-particle size for this flow regime, as opposed to the constant value previously used.

Keywords: Correction function, sedimentation velocity, micro particles.

\section{INTRODUCCIÓN}

La velocidad de sedimentación de las partículas presentes en una suspensión sufre una caída monótona de su valor, a medida que su concentración volumétrica aumenta. El origen de este fenómeno se encuentra en la variación que sufre el comportamiento de las fuerzas hidrodinámicas, osmóticas y electroquímicas entre las partículas a medida que su concentración aumenta. Este fenómeno depende, además, del tamaño de las partículas presentes en la suspensión, ya que para suspensiones conformadas por partículas cuyo diámetro medio sea mayor a $100 \mu \mathrm{m}$, las fuerzas osmóticas y electroquímicas se pueden despreciar [1-4, 6-9, 12-19].

A este respecto es conveniente indicar que las fuerzas hidrodinámicas tienen su origen en la formación, ya sea puentes hidráulicos entre partículas y/o por el arrastre o la succión que las partículas de mayor diámetro realizan

\footnotetext{
1 Universidad de Talca. Departamento de Tecnologías Industriales. E-mail: gsalinas@utalca.cl

2 Universidad de Talca. Departamento de Ciencias de Ingeniería. E-mail: iruiz@utalca.cl

3 Technische Universität Dresden. Arbeitgruppe für Verfahrenstechnik und Umwelttechnik. E-mail: mechanvt@rcs.urz.tu-dresden.de
} 
sobre las de menor diámetro y/o por el impulso que las partículas que desarrollan mayor velocidad tienen sobre otras de menor velocidad, lo que las puede desviar de sus trayectorias originales $[3,4,7,12,17$ y 20$]$. A lo anterior, se suma el efecto dominante que tienen las fuerzas de superficie tipo electroquímico entre las partículas, las que son definidas a través del potencial Zeta o carga electroestática superficial de las partículas, estas fuerzas son relevantes en el caso de micropartículas, ya que las superficies expuestas al electrolito, que en este caso es el fluido, son muy importantes, dada la gran superficie expuesta. Estas fuerzas pueden modelarse a través de la teoría DLVO, desarrollada por Derjarin, Landau, Verwey y Overbeck [1, 17 y 20]. Finalmente, las fuerzas de tipo osmótica surgen a partir del movimiento Browniano de las micro partículas, las que tienden a tratar de equilibrar su concentración entre distintas zonas de la suspensión. Esto produce un proceso de transporte de micropartículas, que va desde las zonas de la suspensión de mayor concentración a aquellas de menor concentración. Este mecanismo de transporte da lugar a la aparición de una presión osmótica, la que se incrementa a medida que la concentración volumétrica de partículas en la suspensión aumenta y su tamaño disminuye, lo que se traduce en una reducción de la velocidad de sedimentación de las micro partículas. Los modelos más tradicionales que se aplican para analizar este fenómeno son los planteados por Hall [11] y Mason y Weawer [13].

Los efectos del incremento relativo de la magnitud de fuerzas hidrodinámicas, osmóticas y electroquímicas que se presentan por la interacción que las distintas partículas que se sumergen simultáneamente tienen entre sí, se manifiesta en una variación del valor de la velocidad de sedimentación efectiva o de sumergimiento de un colectivo de partículas en un fluido viscoso, respecto a la velocidad de sumergimiento que tendría una partícula única de igual diámetro medio que las existentes en el colectivo. Dado el tamaño de orden micrométrico del sistema de partículas, estos efectos se aprecian de mejor manera en el valor que alcanza la velocidad terminal de sedimentación o sumergimiento, cuando la suma de fuerzas hidráulicas que actúan sobre la partícula se equilibran, con lo que ésta alcanza una velocidad de sumergimiento constante.

De lo anterior se desprende que el comportamiento de la velocidad de sedimentación para suspensiones formadas con micro partículas es un problema de cierta dificultad, cuya solución ha sido abordada, a partir de diversos modelos fisicoquímicos relativamente complejos, los que pretenden entregar una solución de cierto carácter general a la problemática. Destacan en ellos los trabajos de Einstein [8] y la tendencia actual de emplear técnicas numéricas para la solución de las complejas ecuaciones que relacionan las distintas variables involucradas [3, 5 y 19$]$.

Una segunda aproximación a la solución del problema es clasificar el comportamiento de la reducción de velocidad de sedimentación a partir de pocos parámetros, tales como el tamaño de partícula, el valor del número de Reynolds del escurrimiento, la concentración volumétrica de partículas y ciertos exponentes, a los que se agrega la velocidad terminal de sedimentación que alcanza una partícula única de igual tamaño medio que las presentes en la suspensión. Esto permite generar un conjunto de ecuaciones de carácter semiempírico que son válidas para distintos rangos de tamaño de micropartículas, en la forma de funciones de corrección, que son valores que de acuerdo a la situación que se analice, plantean un factor que multiplicado por la velocidad terminal de sedimentación permite determinar la velocidad efectiva de sedimentación del colectivo de partículas $[1,2,4,6,14$, 18 y 19]. Este tipo de solución se constituye en una buena alternativa al momento de enfrentar un problema práctico en ingeniería de procesos. Es por ello que su uso sea tan extendido. De facto, para la mayoría de las aplicaciones industriales estas ecuaciones satisfacen adecuadamente las necesidades de diseño y medición. De las distintas funciones de corrección de la velocidad de sedimentación que han sido propuestas, la que tiene mayor aceptación es la que fue propuesta por Richardson y Zaki en 1954 [14], ya que es la que, actualmente, más se utiliza en términos prácticos, en el diseño de sistemas de sedimentación, tales como los equipos de medición de distribución de tamaño de partículas y muchas otras aplicaciones [9, 15-17 y 20].

El planteamiento matemático de la función de corrección de Richardson y Zaki [14] se realiza a partir de la denominada velocidad terminal de sedimentación, que es la velocidad con la cual una partícula alcanza un equilibrio dinámico entre su peso propio, la fuerza de flotación y la fuerza de arrastre viscoso, de manera que su velocidad de sumergimiento se vuelve constante y es posible evaluarla a partir de la fuerza de arrastre viscoso, donde el coeficiente de arrastre se determina a partir de la ecuaciones de Stokes o de Martín o de Newton, según el número de Reynolds que presente el escurrimiento [12, 17 y 20]. Luego, la forma general de la función de corrección de Richardson y Zaki [14] es presentada en la ecuación (1).

$$
F c=\frac{u_{\text {Sedimentación }}}{u_{s}}=\left(1-c_{v}\right)^{n}
$$

Donde:

$$
\begin{array}{ll}
F c & \text { Factor de corrección de Richardson y } \\
& \text { Zaki } \\
\mathrm{u}_{\text {Sedimentación }} & \text { Velocidad efectiva de sedimentación del } \\
& \text { colectivo de partículas }
\end{array}
$$




$$
\begin{array}{ll}
u_{s} & \begin{array}{l}
\text { Velocidad teórica de sedimentación de la } \\
\text { partícula de tamaño medio }
\end{array} \\
c_{v} & \begin{array}{l}
\text { Concentración volumétrica del colectivo } \\
\text { de partículas }
\end{array}
\end{array}
$$

Los trabajos de Richardson y Zaki [14] mostraron que existía una dependencia entre el valor del número de Reynolds alcanzado por el escurrimiento y el valor del exponente de la función de corrección, lo que se muestra en la tabla 1 .

Tabla 1. Exponentes de la función de Richardson y Zaki [14].

\begin{tabular}{|l|l|}
\hline \multicolumn{1}{|c|}{ Rango de número de Reynolds } & Exponente $\mathbf{n}$ \\
\hline $\operatorname{Re} \leq 0,2$ & 4,65 \\
\hline $0,2<\operatorname{Re}<1$ & $4,35 \cdot \mathrm{Re}^{-0,03}$ \\
\hline $1<\operatorname{Re}<500$ & $4,45 \cdot \mathrm{Re}^{-0,1}$ \\
\hline $500<\operatorname{Re}<300000$ & 2,39 \\
\hline
\end{tabular}

A su vez, la velocidad de sedimentación teórica de la partícula puede calcularse de la siguiente manera [10]:

$$
u_{s}=\frac{\left(\rho_{p}-\rho_{f}\right) \cdot x^{2} \cdot z \cdot g}{18 \cdot \mu}
$$

Donde:

$$
\begin{array}{ll}
\rho_{\mathrm{p}} & \text { Densidad media de la partícula } \\
\rho_{\mathrm{f}} & \text { Densidad media del fluido } \\
\mathrm{x} & \text { Diámetro medio del colectivo de partículas } \\
\mathrm{z} & \text { Factor de aceleración } \\
\mathrm{g} & \text { Aceleración de gravedad } \\
\mu & \text { Viscosidad dinámica del fluido }
\end{array}
$$

Por su lado, el número de Reynolds para el caso de partículas esféricas está definido como:

$$
\operatorname{Re}=\frac{x \cdot \rho_{f} \cdot u_{\text {Sedimentación }}}{\mu}
$$

Donde:

\section{Re Número de Reynolds}

Como se aprecia, el valor del exponente de la función de corrección de Richardson y Zaki [14] es empleado para el caso de las partículas que sedimentan en regímenes de escurrimiento laminares definidos a través de números de Reynolds menores a 0,25 es $n=4,65$, sin importar el tamaño de éstas y que corresponde al caso de partículas de tamaño micrométrico.
El presente trabajo muestra que la dependencia entre el valor del exponente de la función de corrección de Richardson y Zaki [14] se mantiene a medida que el tamaño de las partículas que sedimentan disminuye y, por ende, su valor de número de Reynolds, lo que se traduce en que el exponente de la función de corrección de Richardson y Zaki [14] adopta valores mayores al indicado anteriormente. Este comportamiento fue posible evaluarlo a partir de un trabajo de carácter experimental que fue desarrollado con micropartículas calibradas de óxido de silicio $\left(\mathrm{SiO}_{2}\right)$, las que fueron suspendidas en agua con hidróxido de potasio $(\mathrm{KOH})$, el que se empleó como agente antifloculante, siguiendo la norma de ensayo de sedimentación DIN 66111 [10].

\section{TRABAJO EXPERIMENTAL}

Las micropartículas utilizadas para este trabajo fueron del tipo estándares calibrados comerciales, proveídas por la empresa Geltech Inc. Químicamente, estas micropartículas son dióxido de silicio amorfo $\left(\mathrm{SiO}_{2}\right)$, de forma esférica y rígidas, con una densidad media del orden de $2.135 \mathrm{~kg} / \mathrm{m}^{3}$, con diámetros nominales de $1,5 \mu \mathrm{m}$ y 1,0 $\mu \mathrm{m}$. La tabla 2 presenta las principales características del material de referencia. Por su lado, las figuras $1 \mathrm{y}$ 2 muestran fotografías que fueron obtenidas durante los ensayos mediante un microscopio electrónico Zeiss Gemini 942, donde se muestra tanto la forma como el tamaño de las micropartículas de Geltech utilizadas en los ensayos.

Tabla 2. Datos técnicos del material de referencia.

\begin{tabular}{|c|c|c|}
\hline $\begin{array}{c}\text { Diámetro } \\
\text { nominal }\end{array}$ & $\begin{array}{c}\text { Código del } \\
\text { material }\end{array}$ & $\begin{array}{c}\text { Fracción } \\
50 \%\end{array}$ \\
\hline $1,5 \mu \mathrm{m}$ & $\mathrm{S} 1501$ & $1,39 \mu \mathrm{m}$ \\
\hline $1,0 \mu \mathrm{m}$ & $\mathrm{S} 1001$ & $1,06 \mu \mathrm{m}$ \\
\hline
\end{tabular}

En cuanto a las suspensiones, éstas se realizaron con agua tridestilada, desionizada y filtrada, a la cual se le adicionó hidróxido de potasio $(\mathrm{KOH})$, a fin de que alcanzara un valor de índice de acidez $(\mathrm{pH})$ del orden de 9,2. Esto permite alcanzar un potencial Zeta, el que, dependiendo del tamaño de las partículas, sea del orden -40 a $-60 \mathrm{mV}$. Esto se realiza a fin de asegurar la estabilidad de la suspensión, lo que permite reducir la formación de flóculos y aglomerados. 


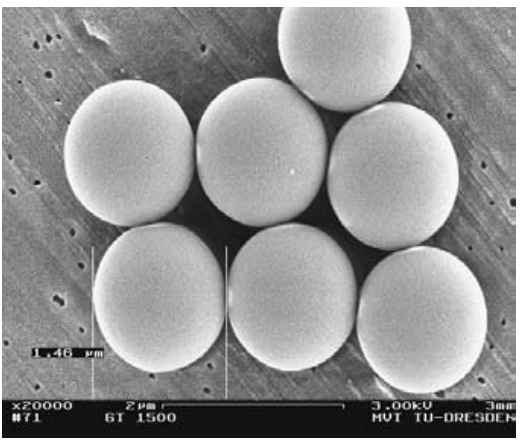

Figura 1. Fotografía de Geltech S1501.

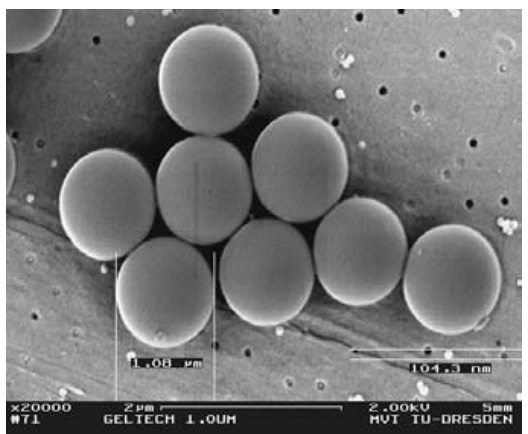

Figura 2. Fotografía de Geltech S1001.

Las suspensiones ensayadas se sometieron a una cuidadosa preparación siguiendo la Norma DIN 66111 [10], a fin de evitar su contaminación por micropartículas extrañas a las que conforman la suspensión de trabajo. Por su lado, el procedimiento de dispersión fue mediante agitación mecánica a alta velocidad y la aplicación de ultrasonido.

Una vez elaborada la suspensión, se procedió a verificar su estado mediante análisis de las imágenes aportadas por el microscopio electrónico Zeiss Gemini 942, las que no revelaron la presencia de partículas extrañas en las suspensiones a ensayarse. La determinación de la distribución de tamaño de partícula se realizó mediante espectrometría láser con un equipo Helos Fa. Sympatec, cuyos resultados se presentan en la figura 3. Estos mostraron también que la dispersión del material particulado fue la adecuada y confirmaron la ausencia de contaminación por partículas extrañas.

En cuanto a la centrífuga analítica empleada para desarrollar los ensayos, ésta fue una máquina LUMiFuge 114, fabricada por L.U.M. GmbH, donde se utilizaron cubetas del tipo rectangular de polystyrol de $3 \times 7 \mathrm{~mm}^{2}$. El análisis de imágenes y la determinación de la velocidad de sedimentación se realizó mediante el software LUM View v. 2.0, que es provisto por la misma firma. Los principales datos técnicos del equipo se presentan en la tabla 3 .

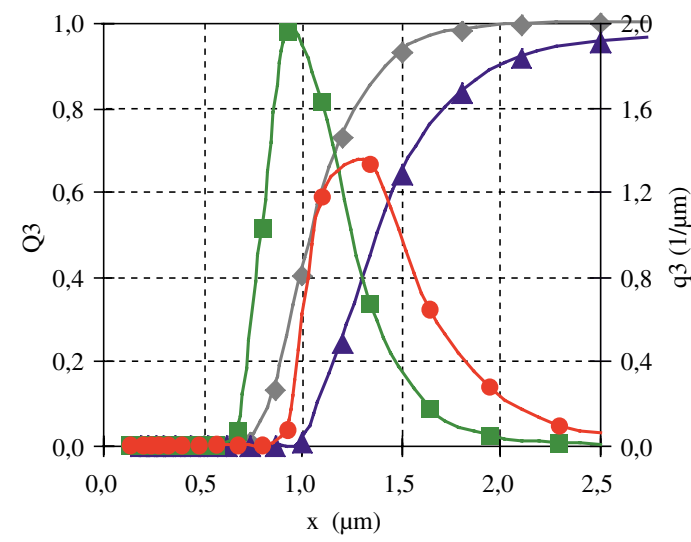

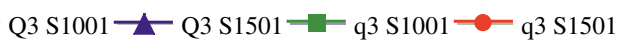

Figura 3. Distribución de tamaño de partícula para Geltech S1001 y Geltech S1501.

Tabla 3. Datos técnicos de la centrífuga analítica LUMiFuge 114.

\begin{tabular}{|c|c|}
\hline Tipo de equipo & $\begin{array}{c}\text { Sistema de análisis de } \\
\text { suspensiones LUMiFuge }^{\mathrm{TM}}\end{array}$ \\
\hline Fabricante & L.U.M. GmbH \\
\hline Intervalo de medida & $10 s-600 s$ \\
\hline Velocidad de centrifugación & $300-3.000 \mathrm{rpm}$ \\
\hline Fuente de luz & nir-led \\
\hline $\begin{array}{l}\text { Longitud de onda en el } \\
\text { espectro BW } 50 \%\end{array}$ & $880 \mathrm{~nm}, 80 \mathrm{~nm}$ \\
\hline Fotosensor & CCD sensor de una fila \\
\hline Número de cubetas & 8 \\
\hline Cubetas & $\begin{array}{l}\text { Rectangulares de polystyrol, } \\
3 \times 7 \mathrm{~mm}^{2} \\
\text { Cilíndricas de vidrio, } \\
\emptyset=11,5 \mathrm{~mm} \\
\text { Cubetas de precisión óptica } \\
\left(1 \times 10 \mathrm{~mm}^{2} \text { y } 2 \times 10 \mathrm{~mm}^{2}\right) \\
\end{array}$ \\
\hline Volumen de prueba & $\begin{array}{l}0,5 \mathrm{~cm}^{3} ; 2 \mathrm{~cm}^{3} ; 0,15 \mathrm{~cm}^{3} \\
0,3 \mathrm{~cm}^{3}\end{array}$ \\
\hline $\begin{array}{l}\text { Rango de concentraciones } \\
\text { volumétricas }\end{array}$ & $1 \%$ hasta $90 \%$ \\
\hline $\begin{array}{l}\text { Rango de tamaños de } \\
\text { partículas }\end{array}$ & $50 \mathrm{~nm}-100.000 \mathrm{~nm}$ \\
\hline $\begin{array}{l}\text { Rango de densidad de } \\
\text { partículas }\end{array}$ & $0,01 \mathrm{~g} / \mathrm{cm}^{3}-10 \mathrm{~g} / \mathrm{cm}^{3}$ \\
\hline $\begin{array}{l}\text { Rango de velocidades de } \\
\text { sedimentación }\end{array}$ & $1 \mathrm{~nm} / \mathrm{s}-750.000 \mathrm{~nm} / \mathrm{s}$ \\
\hline
\end{tabular}

Como ya se mencionó, la norma utilizada para la planificación, desarrollo y análisis de estos ensayos fue la DIN 66111, 
relativa a Métodos de sedimentación y se aplica desde 1989 [10]. Esta norma específica tanto los materiales a emplearse en los ensayos, esto es el fluido, las partículas y el agente dispersante, así como los procedimientos de ensayo, las condiciones ambientales y la forma de representar los resultados de las mediciones de distribución de tamaño de partículas alcanzados. Las figuras siguientes muestran las distribuciones de tamaño de partículas para las diversas suspensiones analizadas según el material de referencia empleado, las que se obtuvieron de las suspensiones ensayadas a través de un espectrómetro láser Helos Fa. Sympatec.

La metodología de preparación de las muestras, así como el rango de la medición y el análisis de los datos suministrados por la centrífuga analítica LUMiFuge 114 son analizados en detalle por [9 y 15], de manera que se asegura la calidad de los datos obtenidos.

\section{RESULTADOS Y DISCUSIÓN}

Los ensayos se realizaron en el Laboratorio de Partículas del Grupo de Trabajo de Técnicas de Procedimientos Mecánicos de la Universidad Técnica de Dresden (Arbeitgruppe für mechanische Verfahrenstechnik an der Technische Universität Dresden) y mostraron la tendencia de la velocidad efectiva de sedimentación respecto de la concentración volumétrica de micropartículas presentes en la suspensión definida por la forma genérica de la función de corrección empleada por Richardson y Zaki [14], pero discrepan en el valor del exponente de la función, como lo muestra la figura 4, donde se presenta la contrastación de los valores experimentales obtenidos con la función de corrección en que se emplea el exponente tradicional $(n=4,65)$, para escurrimientos con número de Reynolds menores a 0,25. Cabe indicar que los valores del número de Reynolds para los escurrimientos estudiados fueron en todos estos casos menores a 0,001 .

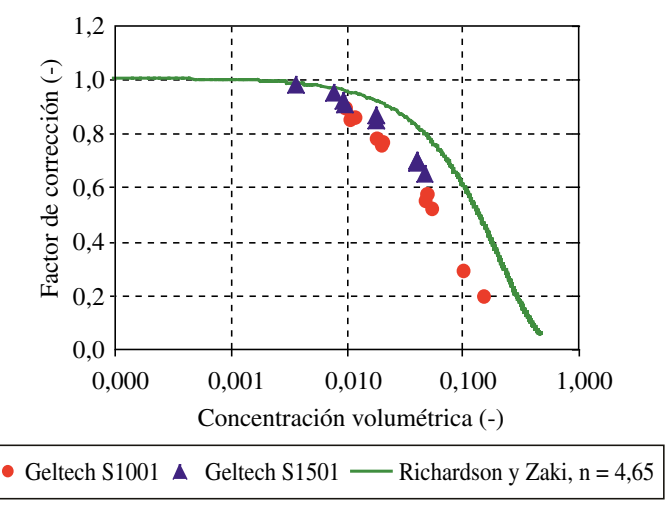

Figura 4. Comparación entre valores experimentales y la función de Richardson y Kaki.
Este comportamiento es esperable, ya que a medida que el tamaño de las micropartículas que conforman la suspensión disminuye, mayor será la cantidad de micropartículas existentes en ésta a igualdad de concentración volumétrica, lo que produce varios efectos, entre los que destaca el hecho que tanto la masa como el volumen de las micropartículas disminuyen, a medida que se reduce el tamaño de éstas, ya que el volumen de una esfera es función directa del diámetro elevado a tres. Esto conduce a que las fuerzas generadas por la acción de un campo mecánico de fuerzas, como el gravitatorio o centrífugo, disminuyan siguiendo la raíz cúbica, mientras que las fuerzas de origen electroquímico se incrementen, ya que el área expuesta por las partículas aumenta al cuadrado. De igual modo, debe considerarse que la fuerza engendrada por la difusión osmótica, que sigue una función exponencial, conduce a que la magnitud relativa de las fuerzas mecánicas disminuya notoriamente frente a las fuerzas electroestáticas y las de origen osmótico. Todo esto se traduce en una reducción de la velocidad de sedimentación del conjunto de micropartículas. Una explicación más amplia del efecto del incremento de las fuerzas electroquímicas se obtiene del hecho que al existir una mayor cantidad de micro partículas en la suspensión por efecto de su disminución de tamaño conduce a que la superficie de las micro partículas en contacto con el fluido -el que de hecho es un electrolito- aumente, lo que implica que las fuerzas electroestáticas, de van der Waals y de atracción de Born, representadas a través del potencial Zeta, aumenten siguiendo lo indicado por la teoría DLVO [17, 20], lo que conduce a un relevante aumento de las fuerzas de repulsión entre las micropartículas adyacentes y con ello la formación de una suerte de red dinámica de micro partículas que impide su sedimentación individual y como colectivo [4-6, 8, 14-18 y 20].

Por su lado, la difusión osmótica de partículas implica la aparición de una fuerza que impulsa el movimiento de las partículas desde la región de mayor concentración de éstas hacia la región de la suspensión donde se presente la menor concentración. Estas regiones coinciden con las regiones adyacentes al sedimento y al espejo de separación entre la suspensión y el fluido claro o sin presencia de partículas. Dado que esta fuerza se dirige en el sentido opuesto de la dirección de sedimentación definida por efecto del campo de fuerzas mecánico, necesariamente se traduce en una reducción de la velocidad de sedimentación efectiva. La importancia de este fenómeno se ve incrementada a medida que el tamaño de las partículas disminuye, ya que tiene como su origen en el movimiento browniano, que es más relevante para el caso de partículas de menor tamaño [2, 11 y 13$]$. 
Para determinar los nuevos valores que toma el exponente de la función de corrección, se procedió a graficar los valores experimentales obtenidos del factor de corrección en función de la concentración volumétrica, en un diagrama doble logarítmico. Luego, se procedió a ajustar a estos valores aplicando una recta como línea de tendencia. Este gráfico se presenta en la figura 5. De las ecuaciones que se ajustan a los valores fue posible evaluar el exponente de la función de corrección de velocidad para ambos casos. Donde para S1510 fue 9,15 $(n=9,15)$, con un coeficiente de correlación 0,996 $(\mathrm{R}=0,996)$. Mientras que para S1001, el exponente de la función de corrección fue 11,31 $(\mathrm{n}=11,31)$ y su coeficiente de correlación $0,992(\mathrm{R}=0,992)$.

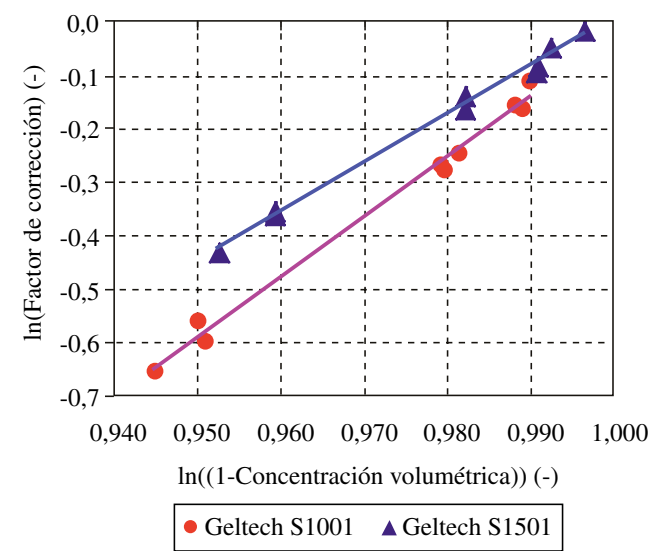

Figura 5. Comportamiento de los exponentes de la función de reducción de los resultados experimentales.

El hecho de emplear exponentes con un mayor valor en la función de Richardson y Zaki [14], conduce a que el nuevo valor que toma el factor de corrección de esta función describe de mejor manera a estos fenómenos, mostrando que la reducción de la velocidad efectiva de sedimentación respecto de la velocidad teórica de sedimentación, es mayor mientras menor sea el tamaño de las micro partículas que conforman la suspensión. Como se muestra en la figura 6, donde se presenta la contrastación de valores del exponente en el orden de los determinados en el trabajo experimental con la forma genérica de la función de Richardson y Zaki [14], demostrando de paso la directa relación que existe entre el valor que toma el exponente con el tamaño de las micro partículas que sedimentan.

Cabe indicar que al realizarse los experimentos de acuerdo a la norma DIN 66111, el utilizar un material de referencia calibrado y equipos de control como el microscopio electrónico y el espectrómetro láser, permite tener confianza en los valores experimentales alcanzados, ya que estos mostraron un muy buen nivel de reproducibilidad, considerando que se realizó una gran cantidad de ensayos y suspensiones, lo que se vio facilitado al emplear la centrífuga analítica como la LUMiFuge 114, que permitía realizar ocho (8) ensayos simultáneos.

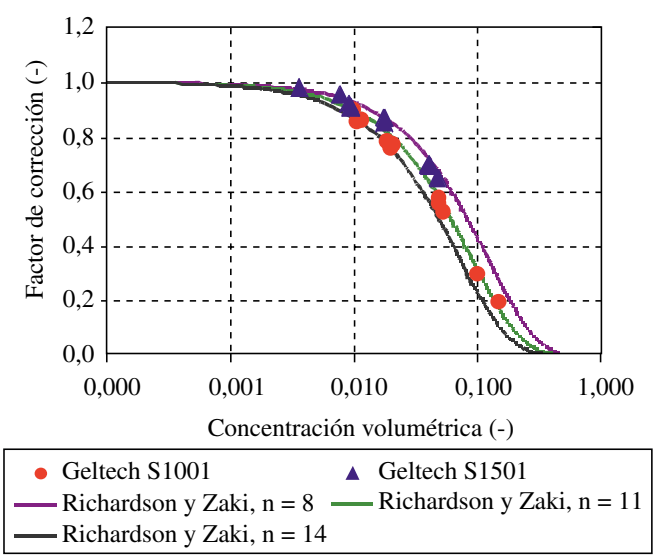

Figura 6. Comparación entre valores experimentales y la función de Richardson y Zaki para distintos exponentes.

\section{CONCLUSIONES}

De este trabajo puede concluirse que los cálculos de velocidad de sedimentación de micropartículas que empleen la función de corrección de Richardson y Zaki [14] con el exponente convencional $(n=4,65)$, muestran significativas diferencias en su predicción del valor de la velocidad de sedimentación respecto de la que realmente se presenta, para el caso de procesos que involucren partículas de tamaño micrométrico. Esto conduce a considerar la necesidad de subdividir la tabla de clasificación en la zona de escurrimiento laminar, a fin de que se consideren los fenómenos asociados a partículas de orden micrométrico e introducir así en esta subdivisión nuevos valores para el exponente, a fin de incrementar la precisión y eficiencia de los diseños prácticos de aquellos equipos que emplean a la sedimentación como su principio de operación.

El considerar un valor para el exponente de la función de corrección de Richardson y Zaki [14] del orden de 11 $(\mathrm{n}=11)$, para partículas con un tamaño medio menor a $1,0 \mu \mathrm{m}$, resultaría adecuado, ya que representa de mejor manera la fenología de la sedimentación de conjuntos o colectivos de micro partículas de dicho tamaño.

Otros trabajos desarrollados por los autores del presente artículo para partículas cuyos diámetros se 
encuentran en el rango submicrométrico no coloidal, muestran que el exponente de la función de corrección de Richardson y Zaki [14] sigue la misma tendencia a aumentar su valor, el que para el caso de partículas de diámetro medio de $0,2 \mu \mathrm{m}$, alcanza una cifra del orden de $16[1,2]$.

Los trabajos que actualmente desarrollan los autores del presente artículo se realizan con partículas de orden de tamaño submicrométrico no coloidales, donde los resultados preliminares muestran no sólo que el valor propuesto por Richardson y Zaki [14] debe ser revisado para este tamaño de partículas. Además, muestran que las bases de algunos de los modelos teóricos elaborados deben ser revisados, ya que al incrementarse el valor del espesor de la capa iónica en torno a las partículas, no sólo influyó en los parámetros de estabilidad de la suspensión, sino que también en la estructura que adoptan las partículas en la suspensión, durante el proceso de sedimentación $\mathrm{y}$, por ende, en los fenómenos hidrodinámicos que se generan.

\section{REFERENCIAS}

[1] F. Babick, M. Stintz and G. Salinas-Salas, "Sedimentation of Colloidal Particles, Experimental study on the influence of the ionic strength on the hindrance function". 12th IACIS International Conference on Surface and Colloid Science. Beijing, China. 2006.

[2] F. Babick, G. Salinas-Salas, T. Sobisch and D. Lerche. "Particle interactions in dispersions of micro and microparticles". PARTEC 2007 Word Congress on Particle Technology. Nürnberg, Germany. 2007.

[3] E. Barnea and J. Mizrahi. "A generalized approach to the fluid dynamics of particulate systems". The Chemical Engineering Journal. Vol. 5, pp. 171-189. 1973.

[4] G.K. Batchelor. "Sedimentation in a dilute dispersion of spheres". Journal Fluid Mechanics. Vol. 119, pp. 379-408. 1982.

[5] R. Bürger, F. Concha, K.K. Fjelde and K. Karsten Hvistendahl. "Numerical simulation of the settling of polydisperse suspensions of spheres". Power Technology. Vol. 113, pp. 30-54. 2000.

[6] P.Y. Cheng and H.K. Schachman. "Studies on the validity of the Einstein viscosity law and Stokes law of sedimentation". Journal Polymer Sci. 16, pp. 19-30. 1955.

[7] R.H. Davis and A. Acrivos. "Sedimentation of noncolloided particles at low Reynolds numbers". Ann. Rev. Fluid Mech. 17, pp. 91-118. 1985.

[8] A. Einstein. "Eine neue Bestimmung der Moleküldimensionen". Annalen der Physik IV Folge 19, pp. 289-306. 1906.

[9] D. Frömer. "Experimentelle und theoretische Untersuchungen zum Sedimentationsver-halten von Erythrozyten im Gravitations- und Zentrifugalfeld". Dissertation; Humboldt Universität. Berlin, Deutschland. 1998.

[10] Deutsches Institut für Normung e. V. DIN. Norme 66111 Über Sedimentationsmetho- den, pp. 61-63. 1989.

[11] K. R. Hall. "Another hard-sphere equation of state". J. Chem. Phys. 57, pp. 2252-2254. 1992.

[12] B. Hoffmann and K. Husemann. "Sinkgeschwindigkeit kugelförmiger Teil-chen in Suspension bei laminar bzw. turbulenter UmströmungExakte Lösung der Bewegungsgleichung”. Chem. Ing. Techn. Vol. $75 \mathrm{~N}^{\mathrm{o}} 3$, pp. 757-763. 2003.

[13] M. Mason and W. Weaver. "The settling of small particles in a fluid". Phys. Rev. Vol. 2, pp. 412-426. 1924.

[14] J.F. Richardson and W.N. Zaki. "Sedimentation and Fluidisation. Part I". Trans. Inst. Chem. Eng., pp. 35-53. 1954.

[15] S. Ripperger, G. Salinas-Salas, M. Stintz and B. Bessely. "Untersuchungen der Sedimentation von microskaligen Partikeln im Zentrifugalfeld". Filtrieren \& Separieren. N ${ }^{\circ}$ 5, pp. 220-238. 2002.

[16] G. Salinas-Salas, M. Stintz y B. Wessely. "Construcción y calibración de una celda de sedimentación gravimétrica para la medición fotométrica de la distribución de tamaño de micropartículas". Rev. Fac. Ing. - Univ. Tarapacá. Vol. 13 No 2, pp. 39-49. 2005. 
[17] H. Schubert, E. Heidenreich, F. Liepe and T. Neeße. "Mechanische Verfahrenstechnik". Deutscher Verlag für Grundstoffindustrie. 1990.

[18] H. Steinour. "Rate of sedimentation: Non-flocculated suspensions of uniform spheres". Ind. Eng. Chem. Vol. $36 \mathrm{~N}^{\circ}$ 7, pp. 168-624. 1944.
[19] M. Ungarisch. "On two-phase flow in a rotating boundary layer". Springer-Verlag. Berlin Heidelberg, Germany. 1993.

[20] M. Zogg. "Einführung in die Mechanische Verfahrenstechnik". Teubner Verlag. Auflage: 3. 1993. 